\title{
Heat Diffusion Near Absorbing Centers in Laser Materials
}

\author{
Herbert S. Bennett \\ Institute for Materials Research, National Bureau of Standards, Washington, D.C. 20234
}

(January 19, 1971)

\begin{abstract}
The solutions to the heat diffusion equation for spherical absorbing centers in laser materials are presented. The ratio of the volume specific heats for the absorbing center and the laser host is found to determine three regions of behavior. Series expansions for small times and for very large times also are given in each of the three regions. Rapidly converging representations for the complementary error function of complex arguments are developed in order to evaluate numerically the region for which the volume specific heat ratio is greater than three fourths.
\end{abstract}

Key words: Absorbing center; complementary error function; heat diffusion; laser host.

\section{Introduction}

In a previous paper [1], a model to describe the behavior of absorbing centers in laser materials has been developed. Computing the thermal stresses predicted by this model requires solutions to the heat diffusion equation for the temperature. The mathematical details for solving the heat diffusion equation are omitted in ref. 1 and are reported in the present paper. In particular, solutions to the heat diffusion equation for spherical absorbing centers in laser materials, series expansions of these solutions at the center-host interface when times are sufficiently small and large, and a proof that in the limit of small inclusions the temperature of the center-host interface is independent of the bulk thermal properties of the inclusion are presented. These solutions may be applied also to other diffusion problems such as oxygen diffusion in $\mathrm{TiO}_{2}, \mathrm{Al}_{2} \mathrm{O}_{3}, \mathrm{ZnO}$, and other oxides [2].

When certain physical conditions exist, some solutions to diffusion equations contain error functions for complex arguments. For example, when the volume specific heat of a spherical absorbing center is greater than three fourths times the volume specific heat of the host, the analytic expressions for the temperature in the host contain error functions for complex arguments. This is the case for platinum inclusions in some neodymium doped laser glasses. The oxygen diffusion problem is a second example. When the volume diffusion coefficient is sufficiently greater than the surface exchange coefficient, the analytic expressions for the oxygen concentration profile also contain error functions for complex arguments. This may be the case for oxygen diffusion in zinc oxide [3]. Representations of the error function for complex arguments which yield efficient computer programs are not readily available in the literature and therefore are derived in the appendix.

\section{Mathematical Description of the Model}

The heat diffusion equation and the boundary conditions are summarized in this section.

A spherical absorbing center of radius $r_{0}(\mathrm{~cm})$ is imbedded in a host of infinite extent. The absorbing center and host are initially at a uniform temperature $T_{0}$. An incoming spherical wave of light (laser beam) with an energy flux $\left(\pi r_{0}^{2} E_{L} / 4 \pi r^{2} \tau\right)\left(\mathrm{W} / \mathrm{cm}^{2}\right)$ falls at time $t=0$ upon the absorbing sphere. The quantity $E_{L}$ is the energy density $\left(\mathrm{J} / \mathrm{cm}^{2}\right)$. The quantity $r=|\mathbf{r}|$ is the distance from the origin of the system, which is the center of the sphere. A square-wave light pulse of width $\tau(\mathrm{s})$ is assumed. The sphere absorbs an equivalent energy flux $H(t)\left(\mathrm{W} / \mathrm{cm}^{2}\right)$ uniformly over its entire surface. The energy flux for a square-wave laser pulse is

$$
H(t)=\left\{\begin{array}{l}
0, \quad \text { when } \quad t<0 \quad \text { and } \quad t>\tau, \\
A\left(\lambda, T_{a}\right)\left(E_{L} / 4 \tau\right), \quad \text { when } \quad 0 \leqslant t \leqslant \tau,
\end{array}\right.
$$

where $A\left(\lambda, T_{a}\right)$ is the absorptance at absolute temperature $T_{a}$ for incident radiation having wavelength $\lambda$. The problem is to compute the temperature $\boldsymbol{\Theta}(\mathbf{r}, t)$ of the absorbing center-host system as a function of time $t$ and distance $r=|\mathbf{r}|$.

The heat diffusion equation describes the time and space dependence of the temperature for large times $t$ and large distances $r$; namely, when $r<r_{0}$ it is

$$
(\partial \Theta(\mathbf{r}, t) / \partial t)=a_{c}^{2} \nabla^{2} \Theta(\mathbf{r}, t)
$$

and when $r>r_{0}$ it is

$$
(\partial \Theta(\mathbf{r}, t) / \partial t)=a_{h}^{2} \nabla^{2} \Theta(\mathbf{r}, t) .
$$


The Laplacian operator is denoted by $\nabla^{2}$ and the thermal diffusivity is $a^{2}=(K / \rho C)\left(\mathrm{cm}^{2} / \mathrm{s}\right)$, where $K$ is the thermal conductivity $\left(\mathrm{W} / \mathrm{cm}{ }^{\circ} \mathrm{C}\right), \rho$ is the density $\left(\mathrm{g} / \mathrm{cm}^{3}\right)$, and $C$ is the heat capacity at constant volume $\left(\mathrm{J} / \mathrm{g}{ }^{\circ} \mathrm{C}\right)$. The subscript $c$ refers to the absorbing center and the subscript $h$ refers to the host. The diffusion equations (2) and (3) are valid only when local thermodynamic equilibrium exists. They become suspect for times comparable to the phonon-phonon collision time $\left(\sim 10^{-12} s\right)$ and for distances comparable to atomic dimensions $\left(\sim 10^{-8} \mathrm{~cm}\right)$. The diffusion equations require a statement of the boundary conditions before solutions are uniquely defined. The temperature has the form,

$$
\Theta(\mathbf{r}, t)=\left\{\begin{array}{l}
T_{0}, \quad t \leqslant 0 \\
T_{0}+T(\mathbf{r}, t), \quad t>0
\end{array}\right.
$$

for all values of $\mathbf{r}$. The function $T(\mathbf{r}, t)$ is the temperature for $t>0$ relative to the ambient temperature $T_{0}$.

The boundary conditions are time dependent. The temperature must be finite everywhere.

$T_{c}(\mathbf{r}=0, t)=$ finite and $T_{h}(\mathbf{r}=\infty, t)=$ finite.

The continuity of the temperature across the centerhost interface is

$$
T_{c}\left(\mathbf{r}=r_{0} \hat{r}, t\right)=T_{h}\left(\mathbf{r}=r_{0} \hat{r}, t\right),
$$

where $\hat{r}$ is the radial unit vector. The conservation of heat flow at the interface gives

$$
\begin{aligned}
-K_{c} \nabla T_{c}\left(\mathbf{r}=r_{0} \hat{r}, t\right) \cdot \hat{r} & +H(t) \\
& =-K_{h} \nabla T_{h}\left(\mathbf{r}=r_{0} \hat{r}, t\right) \cdot \hat{r} .
\end{aligned}
$$

Equations (1) through (7) represent the mathematical description of the model for absorbing centers in laser materials. The physics from which these equations arise is stated in ref. [1]. It is most convenient to use the spherical coordinates $r, \theta$, and $\varphi$ because the heat flux $H(t)$ is independent of $\theta$ and $\varphi$. The problem then becomes a spherically symmetric one. Equations (2), (3), and (7) now have their respective forms,

$$
\begin{aligned}
& \frac{\partial T_{c}(r, t)}{\partial t}=a_{c}^{2}\left(\frac{\partial^{2} T_{c}(r, t)}{\partial r^{2}}+\frac{2}{r} \frac{\partial T_{c}(r, t)}{\partial r}\right), \\
& \text { for } r<r_{0}, \\
& \frac{\partial T_{h}(r, t)}{\partial t}=a_{h}^{2}\left(\frac{\partial^{2} T_{h}(r, t)}{\partial r^{2}}+\frac{2}{r} \frac{\partial T_{h}(r, t)}{\partial r}\right), \\
& \text { for } \quad r>r_{0},
\end{aligned}
$$

and

$$
-K_{c} \frac{\partial T_{c}\left(r=r_{0}, t\right)}{\partial t}+H(t)=-K_{h} \frac{\partial T_{h}\left(r=r_{0}, t\right)}{\partial r} .
$$

\section{Laplace Transform Solutions}

Equations (8) and (9) and the initial conditions and boundary conditions (4), (5), (6), and (10) are solved by taking their Laplace transforms. The Laplace transform of the temperature $T(r, t)$ is denoted by

$$
U(r, s)=\int_{0}^{\infty} \exp (-s t) T(r, t) .
$$

The Laplace operator $\mathscr{L}$ is introduced and is defined by $\mathscr{L}\{T(r, t)\}=U(r, s)$.

The Bromwich integral,

$T(r, t)=\operatorname{Lim}_{d \rightarrow \infty} \frac{1}{2 \pi i} \int_{c-i d}^{c+i d} \exp (s t) U(r, s) d s$,

expresses the temperature in terms of the Laplace transform. The real quantity $c$ is chosen to be sufficiently large so that the integral,

$$
\int_{0}^{\infty} \exp (-s t)|T(r, t)| d t,
$$

exists. The inverse Laplace operator $\mathscr{L}^{-1}$ is defined by $\mathscr{L}^{-1}\{U(r, s)\}=T(r, t)$, and is a convenient way to represent the integral in eq (11). The Laplace transform of the energy flux $H(t)$ is denoted by $h(s)$. Taking the Laplace transform of eq (1) yields that

$$
h(s)=(Q / 4 \tau s)\{1-\exp (-s \tau)\},
$$

where

$$
Q=A\left(\lambda, T_{a}\right)\left(E_{L} / 4\right)
$$

The Laplace transforms of eqs (5), (6), (8), (9), and (10) are respectively.

$$
\begin{aligned}
& U_{c}(0)=\text { finite and } U_{h}(\infty)=\text { finite, } \\
& U_{c}\left(z_{c 0}\right)=U_{h}\left(z_{h 0}\right)
\end{aligned}
$$

$$
z_{c}^{2} \frac{d^{2} U_{c}}{d z_{c}^{2}}+2 z_{c} \frac{d U_{c}}{d z_{c}}-z_{c}^{2} U_{c}+\frac{T_{0}(r, 0) z_{c}^{2}}{s}=0 \quad \text { for } \quad r<r_{0}
$$

$$
z_{h}^{2} \frac{d^{2} U_{h}}{d z_{h}^{2}}+2 z_{h} \frac{d U_{h}}{d z_{h}}-z_{h}^{2} U_{h}+\frac{T_{0}(r, 0) z_{h}^{2}}{s}=0 \quad \text { for } \quad r>r_{0}
$$

$$
-K_{c} \frac{s^{1 / 2}}{a_{c}} \frac{d U_{c}\left(z_{c}=z_{c 0}\right)}{d z_{c}}+h(s)=-K_{h} \frac{s^{1 / 2}}{a_{h}} \frac{d U_{h}\left(z_{h}=z_{h 0}\right)}{d z_{h}},
$$


where

$$
\begin{aligned}
& z_{c}=\left(r s^{1 / 2} / a_{c}\right), z_{h}=\left(r s^{1 / 2} / a_{h}\right), z_{c 0}=\left(r_{0} s^{1 / 2} / a_{c}\right) \\
& z_{h 0}=\left(r_{0} s^{1 / 2} / a_{h}\right), U_{c}(r, s) \equiv U_{c}\left(z_{c}\right), \\
& \text { and } \quad U_{h}(r, s) \equiv U_{h}\left(z_{h}\right) \text {. }
\end{aligned}
$$

General solutions to the Laplace transformed diffusion eqs (16) and (17) are

$$
U(z)=B_{1} \frac{\sinh (z)}{z}+B_{2} \frac{\cosh (z)}{z}+\frac{T_{0}}{s} .
$$

Let us for convenience choose the zero of the temperature scale such that $T_{0}=0$. The condition, $U_{c}(0)=$ finite, requires that $B_{2 c}=0$ and gives

$$
U_{c}\left(z_{c}\right)=B_{1 c}\left\{\sinh \left(z_{c}\right) / z_{c}\right\} .
$$

The condition, $U_{h}(\infty)=$ finite, requires that $B_{1 h}+B_{2 h}=0$ and gives

$$
U_{h}\left(z_{h}\right)=B_{2 h}\left\{\exp \left(-z_{h}\right) / z_{h}\right\} .
$$

The continuity condition (15) relates the constants $B_{1 c}$ and $B_{2 h}$; namely,

$$
B_{1 c}=B_{2 h}\left\{z_{c 0} \exp \left(-z_{h 0}\right) / z_{h 0} \sinh \left(z_{c 0}\right)\right\} .
$$

Finally, the conservation of heat flow through the interface, eq (18), and the condition (22) permit us to express the constant $B_{2 h}$ in terms of $r_{0}, K_{c}, a_{c}, K_{h}$, and $a_{h}$; namely,

$$
\begin{gathered}
B_{2 h}=h(s)\left(z_{h 0} / s^{1 / 2}\right) \exp \left(z_{h 0}\right) \times\left[\left(K_{h} / a_{h}\right)\left(1+z_{h 0}^{-1}\right)\right. \\
-\left(K_{c} / a_{c}\right)\left(z_{c 0}^{-1}-\operatorname{coth}\left(z_{c 0}\right)\right]^{-1} .
\end{gathered}
$$

Substituting eq (23) into eq (21) and evaluating the Bromwich contour integral to obtain the temperature of the host $T_{h}(r, t)$ would be a most ambitious task. Two different arguments exist by which eq (21) reduces to a more tractable form. The first argument treats eq $(23)$ in the limit that $z_{c 0} \ll 1$. Then the coth $\left(z_{c 0}\right)$ in eq $(23)$ is approximated by

$$
\operatorname{coth}\left(z_{c 0}\right) \sim z_{c 0}^{-1}+\left(z_{c 0} / 3\right)+\mathscr{O}\left(z_{c 0}^{3}\right)+\ldots .
$$

and eq (21) thereby is approximated by the expression

$U_{h \infty}\left(z_{h}\right)=\lim _{z_{c 0} \rightarrow 0} U_{h}\left(z_{h}\right)=B_{h}(\infty, s)\left\{\exp \left(-z_{h}\right) / z_{h}\right\}$,

where

$$
\begin{aligned}
B_{h}(\infty, s) & =\frac{h(s) z_{h 0} \exp \left(z_{h 0}\right)}{M\left(s^{1 / 2}+b_{+}\right)\left(s^{1 / 2}+b_{-}\right)}, \\
M & =\left(\rho_{c} C_{c} r_{0} / 3\right), \quad b_{+}=b\left\{1 \mp(1-R)^{1 / 2}\right\}, \\
b & =\left(K_{h} / 2 M a_{h}\right), \quad \text { and } \quad R=\left(4 \rho_{c} C_{c} / 3 \rho_{h} C_{h}\right) .
\end{aligned}
$$

The second argument involves simplifying boundary condition (10). Observe that when $K_{c} \gg K_{h}$ the temperature gradients in the absorbing center are much less than those for the host. In the limit $K_{c} \gg K_{h}$ boundary condition (10) becomes

$$
M \frac{d T_{0}\left(r_{0}^{-}, t\right)}{d t}=K_{h} \frac{\partial T_{h}\left(r_{0}^{+}, t\right)}{\partial r}+H(t),
$$

where $r_{0}^{ \pm}=\operatorname{Lim}_{\epsilon \rightarrow 0}\left(r_{0} \pm \epsilon\right)$. The temperature of the sphere becomes spatially uniform; that is, $T_{c}(r, t)=T_{c}=T_{h}\left(r_{0}, t\right)$ whenever its thermal conductivity is much larger than that of the host. The heat flux into the sphere is given by,

$$
\left\{\left(4 \pi r_{0}^{3} / 3\right) \rho_{c} C_{c}\left(d T_{c} / d t\right) / 4 \pi r_{0}^{2}\right\} \equiv M\left(d T_{c} / d t\right) .
$$

Taking the Laplace transform of the conservation of heat flux, eq (26), which is valid when $K_{c} \gg K_{h}$, the author obtains the same expression for the Laplace transform $U_{h}$ as that given in eq (24). Hence, approximation (24) obtains either when $\left|r_{0} s^{1 / 2} / a_{c}\right| \ll 1$ or when $K_{c} \gg K_{h}$. The latter inequality imposes no restriction on $r_{0}$ and $s$ or equivalently on $r_{0}$ and $t$ but the former inequality does impose such restrictions.

The volume specific heat ratio $R=\left(4 \rho_{c} C_{c} / 3 \rho_{h} C_{h}\right)$ determines three regions of behavior. Namely, when $R<1$, the roots $b_{ \pm}$are real and are not equal; when $R=1$, the roots are real and equal; and when $R>1$, the roots are complex and conjugate to one another. The latter is the region for which the arguments of the complementary error functions are complex. In the next Section, analytic expressions for the temperature $T_{h}(r, t)$ in each of these three regions are presented.

\section{Results and Conclusions}

In this section, closed form expressions for $T_{h}(r, t)$ are given for the regions $R<1, R=1$, and $R>1$. Also, series expansions for large and small values of $b t^{1 / 2}$ are derived.

In the limit that $z_{c 0} \ll 1$ or in the limit that $K_{c} \gg K_{h}$, the Laplace transform of the temperature in the host becomes

$$
U_{h \nsim}\left(z_{h}\right)=[1-\exp (-s \tau)] D_{h}(s)
$$

where eqs (13) and (25) require that

$$
D_{h}(s)=\frac{(Q / 4 \tau s) z_{h 0} \exp \left(z_{h 0}-z_{h}\right)}{z_{h} M\left(s^{1 / 2}+b_{+}\right)\left(s^{1 / 2}+b_{-}\right)}
$$

The translation property of Laplace transforms, (eq (29.2.15) of ref. [4]) then permits one to express the temperature in the host in terms of a Bromwich integral, $B_{I}(r, t)$, and the unit step function, $u(t)$; namely, [5]

$$
T_{h}(r, t)=B_{I}(r, t)-u(t-\tau) B_{I}(r, t-\tau) .
$$

where 


$$
B_{I}(r, t)=\operatorname{Lim}_{d \rightarrow \infty} \frac{1}{2 \pi i} \int_{c-i d}^{c+i d} \exp (s t) D_{h}(s) d s,
$$

and the unit step function $u(t)$ is defined by

$$
u(t)=\left\{\begin{array}{l}
0, t<0 \\
\frac{1}{2}, t=0 \\
1, t>0 .
\end{array}\right.
$$

Subsections $4.1,4.2$, and 4.3 of this section contain respectively the evaluation of the Bromwich integral (30) for the cases of $R<1, R=1$, and $R>1$.

$$
\text { 4.1. } R<1
$$

When the volume specific heat ratio $R$ is less than one, then the roots $b_{+}$and $b_{-}$are real and are not equal. The Bromwich integral (30) is evaluated more readily by expressing $D_{h}(s)$ in terms of partial fractions;

$$
D_{h}(s)=P_{<}(r)\left\{d_{+}(s)-d_{-}(s)\right\},
$$

where

$$
P_{<}(r)=\left\{Q r_{0} / 8 \tau \operatorname{Mbr}(1-R)^{1 / 2}\right\},
$$

and

$$
d_{+}(s)=\left[\exp \left\{\left(r_{0}-r\right) s^{1 / 2} / a_{h}\right\} / s\left(s^{1 / 2}+b_{ \pm}\right)\right]
$$

The transform (34) is valid only when $r>r_{0}$. The Bromwich integral (30) is evaluated by applying eq (29.3.89) of ref. [4] to eqs (32) and (34). This evaluation is correct only when $\gamma=\left\{\left(r-r_{0}\right) / a_{h}\right\} \geqslant 0$. It is found found that according to ref. [4],

$$
\begin{gathered}
T_{h}(r, t ; R<1)=P_{<}(r)\left[b _ { + } ^ { - 1 } \left\{F\left(\gamma, b_{+}, t\right)\right.\right. \\
\left.-u(t-\tau) F\left(\gamma, b_{+}, t-\tau\right)\right\}-b_{-}^{-1}\left\{\boldsymbol{F}\left(\gamma, b_{-}, t\right)\right. \\
\left.\left.-u(t-\tau) F\left(\gamma, b_{-}, t-\tau\right)\right\}\right]
\end{gathered}
$$

where $\gamma=\left\{\left(r-r_{0}\right) / a_{h}\right\} \geqslant 0$. The dimensionless function $F(\gamma, b, t)$ contains the complementary error function; namely,

$$
\begin{array}{r}
F(\gamma, b, t)=-\exp \left(\gamma b+b^{2} t\right) \operatorname{erfc}\left(b t^{1 / 2}+\left(\gamma / 2 t^{1 / 2}\right)\right) \\
+\operatorname{erfc}\left(\gamma / 2 t^{1 / 2}\right)
\end{array}
$$

where

$$
\operatorname{erfc}(x)=1-\frac{2}{\pi^{1 / 2}} \int_{0}^{x} \exp \left(-t^{2}\right) d t
$$

\section{2. $R=1$}

When the volume specific heat ratio $R$ equals one, the roots are real and equal; that is,

$$
b_{+}=b_{-}=b=\left(K_{h} / 2 M a_{h}\right)
$$

and the Laplace transform factor $D_{h}(s)$, eq (28), becomes

where

$$
D_{h}(s)=P_{0}(r) d_{0}(s),
$$

and

$$
P_{0}(r)=\left(Q r_{0} / 4 \tau M b^{2} r\right),
$$

$$
d_{0}(s)=b^{2}\left\{\exp \left(-\gamma s^{1 / 2}\right) / s\left(s^{1 / 2}+b\right)^{2}\right\} .
$$

Because the identity,

$$
\begin{aligned}
& -\mathscr{L}^{-1}\left\{\frac{d}{d b}\left(\frac{\exp \left(-\gamma s^{1 / 2}\right)}{s\left(s^{1 / 2}+b\right)}\right)\right\} \\
& =\mathscr{L}^{-1}\left\{\frac{\exp \left(-\gamma s^{1 / 2}\right)}{s\left(s^{1 / 2}+b\right)^{2}}\right\}=-\frac{d}{d b}\left\{b^{-1} F(\gamma, b, t)\right\},
\end{aligned}
$$

exists, the temperature in the host has the form

$T_{h}(r, t ; R=1)=P_{0}(r)\{G(\gamma, b, t)$

where

$$
-u(t-\tau) G(\gamma, b, t-\tau)\},
$$

$$
\begin{aligned}
G(\gamma, b, t)=- & b^{2} \frac{d}{d b}\left\{b^{-1} F(\gamma, b, t)\right\} \\
=(\gamma b+ & \left.2 b^{2} t+1\right) \exp \left(\gamma b+b^{2} t\right) \operatorname{erfc}\left(b t^{1 / 2}\right. \\
& \left.+\left(\gamma / 2 t^{1 / 2}\right)\right)+\operatorname{erfc}\left(\gamma / 2 t^{1 / 2}\right) \\
& -2\left(b t^{1 / 2} / \pi^{1 / 2}\right) \exp \left(-\gamma^{2} / 4 t\right) .
\end{aligned}
$$

\section{3. $R>1$}

When the volume specific heat ratio is greater than one, the roots are complex and conjugate to one another; that is, $\mathrm{b}_{ \pm}=\nu \mp i \mu$ where $\nu=b$ and $\mu=b(R-1)^{1 / 2}$ for $R>1$. Because $b_{-}=b_{+}^{*}$, the Laplace transform factor $D_{h}(s)$ [eq (28)] becomes

$$
D_{h}(s)=P_{>}(r) d_{>}(s)
$$

where

$$
P_{>}(r)=\left(Q r_{0} / 4 \tau M b r \mu\right)
$$

and

$$
d_{>}(s)=\frac{\exp \left(-\gamma s^{1 / 2}\right)}{2 i s}\left\{\frac{1}{s^{1 / 2}+\nu-i \mu}-\frac{1}{s^{1 / 2}+\nu+i \mu}\right\} .
$$

Evaluating the contour integral which results after eq (44) is substituted into eq (30) by the calculus of residues is a most difficult procedure. Because the integrand in eq (37), exp $\left(-t^{2}\right)$, is analytic everywhere in the finite complex plane, the function, $b^{-1} F(\gamma, b, t)$, may be analytically continued to complex values of $b$. The temperature then is computed by taking the imaginary part of the analytic continuation of 
$b^{-1} \boldsymbol{F}(\gamma, b, t)$ for complex values of $b$; namely,

$$
\begin{aligned}
T_{h}(r, t ; R>1)= & P_{>}(r)\{H(\gamma, \nu, \mu, t) \\
& -u(t-\tau) H(\gamma, \nu, \mu, t-\tau)\},
\end{aligned}
$$

where

$$
H(\gamma, \nu, \mu, t)=b \operatorname{Im}\left\{(\nu-i \mu)^{-1} F(\gamma, \nu-i \mu, t)\right\}
$$

The analytic expression for the function $H(\gamma, \nu, \mu, t)$ is rather lengthy. It contains factors related to the real and the imaginary parts of the complementary error function for complex arguments. The error function for complex arguments and procedures for its numerical evaluation are given in Appendix A. Let us introduce the following notation: $\theta=\mu(\gamma+2 \nu t)$, $\rho=\nu t^{1 / 2}+\left(\gamma / 2 t^{1 / 2}\right)$ and $\omega=\mu t^{1 / 2}$. It is shown in Appendix A that the real and the imaginary parts of the complementary error function for complex arguments contain the following integrals:

$\operatorname{sdaw}(\rho, \omega)=\frac{2}{\pi^{1 / 2}} \exp \left(-\omega^{2}\right) \int_{0}^{\omega} \exp \left(\xi^{2}\right) \sin (2 \rho \xi) d \xi$,

and

cdaw $(\rho, \omega)=\frac{2}{\pi^{1 / 2}} \exp \left(-\omega^{2}\right) \int_{0}^{\omega} \exp \left(\xi^{2}\right) \cos (2 \rho \xi) d \xi$.

The expression for $H(\gamma, \nu, \mu, t)$ becomes, in terms of this notation:

$$
\begin{aligned}
& H(\gamma, \nu, \mu, t)=\left\{b /\left(\nu^{2}+\mu^{2}\right)\right\}\left[\mu \operatorname{erfc}\left(\gamma / 2 t^{1 / 2}\right)\right. \\
& -\exp \left(\gamma \nu+\nu^{2} t-\mu^{2} t\right)\{(-\nu \sin \theta+\mu \cos \theta) \\
& \times\left(\operatorname{erfc}(\rho)-\exp \left(\omega^{2}-\rho^{2}\right) \operatorname{sdaw}(\rho, \omega)\right) \\
& \left.\left.+(\nu \cos \theta+\mu \sin \theta) \exp \left(\omega^{2}-\rho^{2}\right) \operatorname{cdaw}(\rho, \omega)\right\}\right] .
\end{aligned}
$$

At first one may question the oscillatory factors, cosine of $\theta$ and sine of $\theta$, which appear in eqs (47) and (48). One does not usually expect such behavior for solutions to a diffusion equation which has no oscillatory boundary conditions or source terms. However, the oscillatory factors in eq (48) are over dampened so that the spatial gradient is always less than zero for all $r \geqslant r_{0}$ [6]. Similar oscillatory factors arise in other diffusion problems. Theoretical solutions for fluid turbulence and for the characteristic lines produced by a moving disc in a uniformly rotating, nonviscous, and incompressible liquid also may contain oscillatory factors [7]. The oscillatory factors in eq (48) exhibit a wavelength $\lambda_{\text {osc }}=\left(2 \pi a_{h} / \mu\right)$, a frequency $\left(1 / \tau_{\text {osc }}\right)=(\mu \nu / \pi)$, and a velocity $\mathbf{v}_{\text {osc }}=-2 \nu a_{h} \hat{r}$. The oscillatory speed, $\left|\mathbf{v}_{\text {osc }}\right|=2 \nu a_{h}$ is less than the speed of sound in the host for all the situations discussed in ref. [1]. If this were not the case then the quasi-static linear thermoelastic theory would be suspect.

\subsection{Expansions for $b t^{1 / 2} \ll 1$}

In order to avoid overflow complications with the computer it is necessary to use series expansions for the functions when they are evaluated at the centerhost interface, $\gamma=0$. These expansions readily show that as $b t^{1 / 2}$ approaches 0 the surface temperature $T_{h}\left(r_{0}, t\right)$ depends only on the properties of the absorbing center. This agrees with the intuitive statement that for sufficiently short times very little heat enters the host. The expansions for the $F, G$, and $H$ functions when $b t^{1 / 2} \ll 1$ are listed now:

$$
\begin{aligned}
F(0, b, t) \sim\left(2 / \pi^{1 / 2}\right) b t^{1 / 2} & -\left(b t^{1 / 2}\right)^{2}+\left(4 / 3 \pi^{1 / 2}\right)\left(b t^{1 / 2}\right)^{3} \\
& +\mathcal{O}\left(b t^{1 / 2}\right)^{4}+\ldots .
\end{aligned}
$$

$G(0, b, t) \sim\left(b t^{1 / 2}\right)^{2}-\left(8 / 3 \pi^{1 / 2}\right)\left(b t^{1 / 2}\right)^{3}$

$$
+\mathcal{O}\left(b t^{1 / 2}\right)^{4}+\ldots .
$$

and

$$
\begin{array}{r}
H(0, \nu, \mu, t) \sim b \mu t\left[1-\left(8 / 3 \pi^{1 / 2}\right) \nu t^{1 / 2}\right. \\
\left.+\mathscr{O}\left(t^{1 / 2}\right)^{2}+\ldots\right]
\end{array}
$$

where $\nu=b$.

Finally, substituting eqs (49), (50), and (51) respectively into eqs (35), (42), and (47) yields when $b t^{1 / 2} \ll 1$ and $t \leqslant \tau$,

$$
T_{h}\left(r_{0}, t\right) \sim(Q t / 4 \tau M) .
$$

The quantity $M$ depends only on the properties of the absorbing center.

\subsection{Asymptotic Expansions for large $b t^{1 / 2} \gg 1$}

In this subsection, the asymptotic expansions for $F$ and $G$ when $b t^{1 / 2} \gg 1$ are listed below. Such expansions for $H$ are not as straightforward as those for $F$ and $G$. In fact, the derivation of a representation for $H$ which converges rapidly when $\nu t^{1 / 2} \gg 1$ is most essential in the development of a computer program to evaluate $T_{h}(r, t)$ for the case in which $R>1$. Representations for $H$ are developed in appendix A. These expansions show that as $r_{0}$ approaches 0 the surface temperature $T_{h}\left(r_{0}, t\right)$ depends only on the absorptance of the interface and the properties of the host and it becomes independent of the other properties of the absorbing center. The asymptotic expansions for the functions $F$ and $G$ when $b t^{1 / 2} \gg 1$ are respectively,

$$
F(0, b, t) \sim 1-\left(1 / \pi^{1 / 2} b t^{1 / 2}\right)+. . .,
$$

and

$$
G(0, b, t) \sim 1-\left(2 / \pi^{1 / 2} b t^{1 / 2}\right)+\mid . . .
$$

Substituting eq (53) into eq (35) gives expressions for the interface temperature when $r_{0}$ is very small; 
namely, if $t \gg \tau$ and $b \tau^{1 / 2} \gg 1$, then

$$
T_{h}\left(r_{0}, t\right) \sim\left(Q r_{0}^{2} / 8 \pi^{1 / 2} a_{h} K_{h} t^{3 / 2}\right),
$$

and if $t=\tau$ and $b \tau^{1 / 2} \gg 1$, then

$$
T_{h}\left(r_{0}, \tau\right) \sim\left(Q r_{0} / 4 \tau K_{h}\right) .
$$

Observe that eqs (55) and (56) do not contain any of the bulk thermal properties of the absorbing center.

The expressions for $T_{h}(r, t)$ given above form the mathematical framework upon which the computer program used to generate the tables of ref. [1] is developed. In addition, several first and second order derivatives of the functions $F, G$, and $H$ with respect to time and space are needed in order to evaluate the temperature for large times and in order to check numerically that the temperature gradient is always negative and that the rate of expansion of the spherc never exceeds the speed of sound in the host. Because closed form expressions for the derivatives are obtained by conceptionally straightforward methods and because these expressions are very lengthy in the case of the $H$ function, the author chooses not to list them here. Once the temperature has been calculated then the optical path length, the stress components, and the stress birefringence near the absorbing center are readily found. These problems have been discussed in detail in ref. [1].

The author thanks W. Gautshi for communicating his results on the efficient computation of the complex error function prior to their publication. He also acknowledges many helpful discussions with A. D. Franklin, A. Kahn, S. M. Wiederhorn, and H. Oser.

\section{Appendix A. Complementary Error Function for Complex Arguments}

A computer program which evaluates the integral,

$$
\int_{r_{0}}^{r} T_{h}(\rho, t) \rho^{2} d \rho, \quad \text { for } \quad R>1,
$$

hundreds of times must incorporate a rapidly converging representation of the complementary error function, if the total computation time is to be reasonable. Several representations of the complementary error function and those representations which are used in the studies on inclusions in laser materials [1] are presented in this Appendix. Computer programs for all of the representations given here have been written in Fortran [8].

Let us begin with the analytic continuation of the complementary error function to complex arguments $z=x-i y$, where $x$ and $y$ are real. The complementary error function for real arguments $z=x$ is defined by

$$
\operatorname{erfc}(x)=1-\frac{2}{\pi^{1 / 2}} \int_{0}^{x} \exp \left(-t^{2}\right) d t
$$

Because the integrand exp $\left(-t^{2}\right)$ is analytic everywhere, the value of the integral is independent of the path along which the variable $t$ follows in going from $t=0$ to $t=z=x-i y$. The following path is chosen:

$$
\begin{aligned}
\operatorname{erfc}(z)=1-\frac{2}{\pi^{1 / 2}} \int_{0}^{x} \exp \left(-t^{2}\right) d t & \\
& -\frac{2}{\pi^{1 / 2}} \int_{x}^{x-i y} \exp \left(-t^{2}\right) d t .
\end{aligned}
$$

Letting $t=x-i s$ in the second integral, the author writes

$$
\begin{aligned}
& \operatorname{erfc}(z)=\operatorname{erfc}(x) \\
&+\frac{2 i}{\pi^{1 / 2}} \exp \left(-x^{2}\right) \int_{0}^{y} \exp \left(s^{2}+2 i x s\right) d s .
\end{aligned}
$$

It is convenient to define

$$
\begin{aligned}
I(x, y) & =\text { cdaw }(x, y)+i \text { sdaw }(x, y) \\
& =\frac{2}{\pi^{1 / 2}} \exp \left(-y^{2}\right) \int_{0}^{y} \exp \left(s^{2}+2 i x s\right) d s,
\end{aligned}
$$

where

cdaw $(x, y)=\operatorname{Re}\{I(x, y)\}$

$=\frac{2}{\pi^{1 / 2}} \exp \left(-y^{2}\right) \int_{0}^{y} \exp \left(s^{2}\right) \cos (2 x s) d s$,

and

sdaw $(x, y)=\operatorname{Im}\{I(x, y)\}$

$$
=\frac{2}{\pi^{1 / 2}} \exp \left(-y^{2}\right) \int_{0}^{y} \exp \left(s^{2}\right) \sin (2 x s) d s .
$$

Inserting these definitions into eq (A2) yields

$$
\begin{aligned}
& \operatorname{erfc}(z)=\operatorname{erfc}(x) \\
& \quad-\exp \left(y^{2}-x^{2}\right)\{\text { sdaw }(x, y)-i \text { cdaw }(x, y)\}
\end{aligned}
$$

where $z=x-i y$. The definition,

$\operatorname{erfs}(z=x-i y)=\operatorname{erfc}(x)-\exp \left(y^{2}-x^{2}\right)$ sdaw $(x, y)$, is introduced.

Many rational approximations for the error function are listed in ref. [4], sections 7.1.25 to 7.1.28, and are readily modified to evaluate exp $\left(x^{2}\right)$ erfc $(x)$. One possibility uses the rational approximation section 7.1.26 of ref. [4]. It is the representation used in ref. [1] whenever $z$ is real and $z=x<2.1$; namely,

$\exp \left(x^{2}\right) \operatorname{erfc}(x)=\sum_{n=1}^{5} a_{n} t^{n}+\exp \left(x^{2}\right) \epsilon(x)$, 
where

$$
t=(1+p x)^{-1} \quad \text { and } \quad|\epsilon(x)| \leqslant \quad 1.5 \times 10^{-7} .
$$

The numerical constants $p, a_{1}, a_{2}, a_{3}, a_{4}$, and $a_{5}$ are given in ref. [4] section 7.1.26. The last term estimates the error and is large for large values of $x$.

Two series expansions for small values of $x$ are

$$
\operatorname{erfc}(x)=1-\frac{2}{\pi^{1 / 2}} \sum_{n=0}^{\infty} \frac{(-1)^{n} x^{2 n+1}}{n !(2 n+1)}
$$

and

$\operatorname{erfc}(x)=1-\frac{2}{\pi^{1 / 2}} \exp \left(-x^{2}\right) \sum_{n=0}^{\infty} \frac{2^{n} x^{2 n+1}}{1 \cdot 3 \ldots(2 n+1)}$.

(A8b)

An asymptotic expansion for large values of $x$ exists; namely,

$\pi^{1 / 2} x \exp \left(x^{2}\right) \operatorname{erfc}(x) \sim 1$

$$
+\sum_{n=1}^{\infty} \frac{(-1)^{n} 1 \cdot 3 \ldots(2 n-1)}{\left(2 x^{2}\right)^{n}}
$$

Equations (A8) and (A9) may be continued analytically to complex values $z=x-i y=r \exp (-i \beta)$, where $r=\left(x^{2}+y^{2}\right)^{1 / 2}$ and $\beta=\tan ^{-1}(y / x)$. The series expansions for small $|z|=r$ becomes

$$
\begin{array}{r}
\cos (2 n \pi s / a)]=\left(a / \pi^{1 / 2}\right)\left[1+2 \sum_{n=1}^{\infty}\right. \\
\left.\exp \left(-n^{2} a^{2}\right) \cosh (2 n s a)\right]
\end{array}
$$

where $a$ is a real positive number, $0<a \leqslant 1$. The constant $a$ is chosen so that

$$
E=2 \sum_{n=1}^{\infty} \exp \left(-n^{2} \pi^{2} / a^{2}\right) \cos (2 n \pi s / a) \ll 1 .
$$

When $a=(1 / 2)$, the term $E$ is of the order of $10^{-17}$ and eq (Al3) becomes an approximate expression for $\exp \left(s^{2}\right) ;$ namely,

$$
\begin{aligned}
& \exp \left(s^{2}\right) \sim\left(a / \pi^{1 / 2}\right) \\
& {\left[1+2 \sum_{n=1}^{\infty} \exp \left(-n^{2} a^{2}\right) \cosh (2 n s a)\right]} \\
& +\mathscr{O}\left(2 \exp \left(-\pi^{2} / a^{2}\right)\right) .
\end{aligned}
$$

The integrations over $s$ in the eqs (A4) and (A5) can be performed in a closed form when approximation (A14) replaces $\exp \left(s^{2}\right)$ in the integrand.

and

$$
\operatorname{erfc}(z)=1-\frac{2}{\pi^{1 / 2}} \sum_{n=0}^{\infty} \frac{(-1)^{n} r^{2 n+1}\{\cos ((2 n+1) \beta)-i \sin ((2 n+1) \beta)\}}{n !(2 n+1)},
$$

$$
\operatorname{erfc}(z)=1-\frac{2 r \exp \left(-x^{2}+y^{2}\right)}{\pi^{1 / 2}} \times \sum_{n=0}^{\infty} \frac{2^{n} r^{2 n}\{\cos ((2 n+1) \beta+2 x y)-i \sin ((2 n+1) \beta+2 x y)\}}{1 \cdot 3 \ldots(2 n+1)} .
$$

The asymptotic expansion for large $|z|$ and for $|\beta|<(3 \pi / 4)$ is

$\operatorname{erfc}(z) \sim \frac{\exp \left(-x^{2}+y^{2}\right)}{\pi^{1 / 2} r}\{\cos (\beta+2 x y)+i \sin (\beta+2 x y)\}$

$$
+\sum_{n=1}^{\infty} \frac{(-1)^{n} 1 \cdot 3 \ldots(2 n-1)}{2^{n} r^{2 n}}\{\cos ((2 n-1) \beta+2 x y)-i \sin ((2 n-1) \beta+2 x y)\}
$$

Representations for the functions cdaw $(x, y)$ and sdaw $(x, y)$ follow from eqs (A6), (A10), and (A11), and (A12).

A corollary of Poisson's formula is the basis for another approximation for the complementary error function. It is more convenient in this case to first approximate cdaw $(x, y)$ and sdaw $(x, y)$. One may derive by means of Poisson's formula (7) the identity, [9]

$\exp \left(s^{2}\right)\left[1+2 \sum_{n=1}^{\infty} \exp \left(-n^{2} \pi^{2} / a^{2}\right)\right.$
The results are

cdaw $(x, y) \sim \frac{2}{\pi^{1 / 2}} \exp \left(-y^{2}\right)$

$$
\begin{gathered}
\left\{\frac{a}{\pi^{1 / 2}} \frac{\sin (2 x y)}{2 x}+\frac{a}{\pi^{1 / 2}} \sum_{n=1}^{\infty} \frac{\exp \left(-n^{2} a^{2}\right)}{\left(n^{2} a^{2}+x^{2}\right)}\right. \\
(n a \sinh (2 n a y) \cos (2 x y) \\
+x \cosh (2 n a y) \sin (2 x y))\},
\end{gathered}
$$




$$
\begin{gathered}
\operatorname{sdaw}(x, y) \sim \frac{2}{\pi^{1 / 2}} \exp \left(-y^{2}\right) \\
\qquad \begin{array}{c}
\frac{a}{\pi^{1 / 2}} \frac{(1-\cos (2 x y))}{2 x}+\frac{a}{\pi} \sum_{n=1}^{\infty} \frac{\exp \left(-n^{2} a^{2}\right)}{\left(n^{2} a^{2}+x^{2}\right)} \\
(x+n a \sinh (2 n a y) \sin (2 x y) \\
-x \cosh (2 n a y) \cos (2 x y))\}
\end{array}
\end{gathered}
$$

Among the above approximations which are valid for large $|z|$ approximations (A15) and (A16) converge most rapidly. However, they are still not as efficient as the algorithm for the real and imaginary parts of the function,

$$
W(z)=\exp \left(-x^{2}\right) \operatorname{erfc}(-i x)
$$

which is given in ref. [10]. The algorithm is based upon the continued fraction representation,

$$
\exp \left(z^{2}\right) \operatorname{erfc}(z)=\frac{1}{\pi^{1 / 2}} \frac{1}{z+} \frac{(1 / 2)}{z+} \frac{1}{z+} \frac{(3 / 2)}{z+} \frac{2}{z+} . .
$$

and efficiently separates the real and imaginary parts of eq (A18).

The complex function $I(x, y)$, eq (A3), can be expressed in terms of the function $W(z)$;

$$
\begin{aligned}
I(x, y)=i \exp & \left(x^{2}-y^{2}\right)\left\{\exp \left(-x^{2}\right) W(i x)\right. \\
- & \left.\exp \left(-(x-i y)^{2}\right) W(i x+y)\right\} .
\end{aligned}
$$

Introducing the notation $W(i x)=W_{1}+i W_{2}$ and $W(i x+y)=W_{3}+i W_{4}$, where $W_{1}, W_{2}, W_{3}$, and $W_{4}$ are real, produces the result

$$
\begin{array}{r}
\operatorname{cdaw}(x, y)=-W_{2} \exp \left(-y^{2}\right)+\left\{W_{4} \cos (2 x y)\right. \\
\left.+W_{3} \sin (2 x y)\right\},
\end{array}
$$

and

$$
\begin{aligned}
\operatorname{sdaw}(x, y)=W_{1} \exp \left(-y^{2}\right) & +\left\{W_{4} \sin (2 x y)\right. \\
& \left.-W_{3} \cos (2 x y)\right\} .
\end{aligned}
$$

A Fortran program based upon the algorithm of ref. [8] to evaluate the real and the imaginary parts of the function $W$ [i.e., $\operatorname{Re}\{W(z)\}$ and $\operatorname{Im}\{W(z)\}]$ has been written.

Computer programs have been written to evaluate all of the above representations for the complementary error function. The prescriptions given below are more efficient than the many others examined during the computations involved in ref. [1]. First consider the case for $\operatorname{Im}\{z\}=0$; that is, $z=x$ and $y=0$. The rational approximation (A7) is used when $0<|x| \leqslant 2.1$ and the continued fraction representation eq (A18) is used when $2.1<|x|<\infty$. This combination is efficient for study of inclusions in laser materials when the volume specific ratio satisfies the inequality $R \leqslant 1$. Next consider the case when $z=x-i y$. The series approximation (A10) is used whenever both $0<|x| \leqslant 0.001$ and $0<|y| \leqslant 0.001$ and the algorithm of ref. [8], is used for all other values in the complex plane. This combination is efficient for the study of inclusions in laser materials when the volume specific heat ratio satisfies the inequality $R>1$.

\section{References and Notes}

[1] Bennett, H. S., Inclusions in Laser Materials, J. Res. Nat. Bur. Stand. (U.S.), 75A (Physics and Chem.), No. 4, 247 (JulyAug. 1971).

[2] Dragoo, A. L., private communication.

[3] Heuer, A., private communication.

[4] Abramowitz, M., and Stegun, I., editors, Handbook of Mathematical Functions (Dover Publications, Inc., New York, 1965), p. 1019.

[5] Whenever $t \gg \tau$, the expression (29) becomes a difference between almost equal numbers. Such a difference must be avoided in a computer program. Whenever $t>10^{5} \tau$, the expression (29) is replaced in the algorithm with the expression

$$
\begin{aligned}
& \operatorname{Lim} \\
& (t / \tau) \rightarrow \infty
\end{aligned} T_{h}(r, t)=\tau\left\{\partial B_{I}(r, t) / \partial t\right\} .
$$

[6] The temperature gradient is negative for all the results reported in ref. [1]; i.e., $\nabla T_{h}<0$. Hence, the second law of thermodynamics is not violated by the model.

[7] Oser, H., Angew, Z., Math. Mech. 38, 27 (1958).

[8] Copies of the Fortran listing are available from the Computer Services Division at the National Bureau of Standards. The listing contains many representations and the user should decide which ones suit his requirements best.

[9] Titchmarsh, E. C., Introduction to the Theory of Fourier Integrals (Oxford Press, Oxford, 1937), pp. 60-64; and Salzer, H. E., Math. Tables and Aid to Comp. 5, 67 (1951).

[10] Gautschi, W., Comm. ACM 12, 635 (1969); and W. Gautschi, SIAM J. Math. Anal., to be published.

(Paper 75A4-667) 